\title{
L'éducation entre reproduction de programmes et formation de soi: l'importance du trajet anthropoformateur
}

\author{
The Education between program reproduction and self-formation: \\ the importance of the anthropoformer path
}

Patrick PAUL ${ }^{1}$

\begin{abstract}
Résumé
Il existe différentes manières de " penser le monde " et de produire du savoir. La compréhension qu'ont les indigènes du monde qui les entoure est par exemple très différente de celle d'un scientifique. Tout être humain se forme grâce à sa propre expérience (savoirs expérientiels) et grâce à un certain nombre de connaissances formelles qui interprètent les phénomènes sélectionnés en donnant un sens à leur lecture. Cet entendement est associé, pour parler un langage académique, au positionnement épistémologique qui permet de les analyser. Ce constat évoque la notion de paradigme (Thomas Kuhn), que l'on peut définir comme une représentation du monde construite par des nécessités socio-historiques, une manière de voir et de penser le plus souvent implicite dans la société mais qui repose cependant sur un fondement parfaitement défini culturellement (un modèle théorique, un courant de pensée).
\end{abstract}

Mots-clés: L'éducation. Épistémologie. Subjectivité.
There are different ways of "thinking the world" and producing knowledge. The understanding that natives have of the world around them is, for example, very different from that of a scientist. Every human being is formed through his own experience (experiential knowledge) and through a certain amount of formal knowledge that interprets the selected phenomena by giving meaning to their reading. This understanding is associated, to speak an academic language, with the epistemological positioning which makes it possible to analyze them. This observation evokes the notion of paradigm (Thomas Kuhn), which can be defined as a representation of the world constructed by socio-historical necessities, a way of seeing and thinking most often implicit in society, but which nevertheless rests on a perfectly culturally defined foundation (a theoretical model, a current of thought).

Keywords: Education. Epistemology. Subjectivity.

1 Graduação em Medicina na Faculdade de Medicina da Universidade Méditerranée dAix-Marseille II (1972), doutorado em Medicina na mesma universidade (1975), mestrado em bioquímica e microbiologia na Faculdade de Ciências da Universidade Paris Diderot Paris VII (1977), mestrado em Antropologia Médica na Faculdade de Medicina de Bobigny-Paris XIII (1999), doutorado em Ciências da Educacão pela Universidade François Rabelais de Tours (2001), França, e Habilité à Diriger des Recherches HDR (equivalente à Livre Docência) em Ciências da Educaçáo na Universidade Rennes II, Haute Bretagne (2004).Membro Expert do Comitê de Organização das Recomendaçóes profissionais sobre o tema da\&quot;L?évaluation des programmes en éducation thérapeutique - 05R12\&quot; [Avaliaçáo dos programas em Educação Terapêutica], Haute Autorité de Santé [Alta Autoridade de Saúde], Paris. Atualmente Professor Visitante da Faculdade de Medicina da Universidade de São Paulo, vinculado ao departamento de Pediatria (2018-2020). E-mail: docppaul@gmail.com

\begin{tabular}{|l|l|l|l|l|l|} 
R. Educ. Públ. & Cuiabá & v. 28 & n. 68 & p. $403-411$ & maio/ago. 2019 \\
\hline
\end{tabular}


Le paradigme aujourd'hui dominant, que l'on dit "scientifique ", apparaît au cours du XIXème siècle et remplace le dualisme (Descartes) apparu au XVIIème siècle. Il se prolonge jusqu'à présent. On le définit comme un monisme matérialiste. Il repose sur deux hypothèses, l'une valorisant l'objectivité (le réel est indépendant des observateurs), l'autre le déterminisme (une cause antérieure ou causalité explique la réalité d'un mode unique, reproductible et objectif. Le réductionnisme méthodologique complète la démarche. Il consiste à dire que la connaissance ressort de la réduction du tout en parties indépendamment de toute subjectivité, ce qui se concrétise par l'apparition des disciplines académiques que nous connaissons. Mais ces clôtures disciplinaires, bien que très efficientes au centre de leur " objet ", se révèlent plus fragiles dans leurs interfaces avec les autres objets disciplinaires. Pour user d'une métaphore, se centrer sur soi rend difficile la communication aux autres.

Mais le paradigme dominant, malgré son efficacité, n'est pas suffisant pour répondre à un certain nombre de défis de la modernité. Car le mouvement scientifique consistant à séparer le tout en parties (en disciplines) répond difficilement au contre-mouvement qui consisterait à réunir les disciplines, à favoriser leur dialogue et leurs interactions. Pour prendre une image, un agro toxique peut être pertinent à utiliser dans le contexte réduit de la culture d'un champ. Mais il peut avoir des conséquences insoupçonnées sur l'environnement global. Ce qui est vrai pour la partie peut avoir un effet contraire sur le tout. Face aux limites de la méthode réductionniste, sont donc apparues, au fur et à mesure du XXème siècle, des tentatives d'articulation : pluri ou multidisciplinarité, puis vers les années 50 l'interdisciplinarité enfin, après les années 70, la transdisciplinarité. Chacune de ces tentatives de dialogue et de réunion entre les disciplines possède une définition précise (P. Paul, 2011) sur laquelle nous ne reviendrons pas.

La transdisciplinarité plus précisément, thème de cette table, possède, avec l'interdisciplinarité, une hypothèse phénoménologique qui reconnaît le sujet et la subjectivité : les propriétés des objets dépendent de la posture de l'observation et de leur analyse, nécessairement plurielle. Car ce qui est " entre " (interdisciplinarité), ou " entre " et " au-delà " (transdisciplinarité) des disciplines est le sujet lui-même. Cette résurrection du sujet s'effectue cependant dans l'interdisciplinarité par un sujet supporté par les seules valeurs biologiques, psychologiques et sociétales alors que la transdisciplinarité offre une vision amplifiée et complexifiée du sujet associé à différents niveaux de réalité, différents degrés du processus d'ontogénèse et différentes formes de rationalité. De même, l'interdisciplinarité n'accueille que les disciplines académiques là où la transdisciplinarité intègre savoirs académiques et non académiques (tacites, expérientiels, traditionnels, spirituels, d'autoformation etc.). 
En éducation, le paradigme actuel s'inscrit dans le réductionnisme et l'objectivité. Il donne priorité aux programmes, aux curriculums, aux parcours de formations, aux évaluations objectives au détriment du sujet et de sa réalité.

Il devient nécessaire de s'ouvrir au questionnement associé aux personnes et à leurs singularités. Autrement dit, de se rouvrir à la question de l'ontologie particulièrement malmenée par le matérialisme et le réductionnisme. Éduquer, certainement, mais pour quoi et pour qui?

Ce questionnement peut apparaître éloigné des préoccupations éducatives actuelles. En même temps, l'éducation, comme de nombreux autres secteurs de la société, traverse une crise grave et une perte importante de sens. Revenir à ce qui la fonde peut se révéler utile afin de favoriser les réorientations. Nous allons, pour éclairer nos propos, revenir à l'étymologie des mots « éducation » ou "pédagogie ":

- Le mot " éducation " provient du latin " educare ${ }^{2}$ ", dont la racine est «dux», " ducis» désignant un chef, un titre de noblesse et, de l'autre, elle avance l'idée ("ducere $)$ de " conduire ».

- Le verbe "ducare " d'où provient "éduquer " (" educare »), signifie en même temps « élever " (l'idée de chef), " nourrir » et " produire ».

- La racine " ex-ducere " implique l'idée de " conduire hors de ", c'est-à-dire d'émanciper, de développer le sens de la responsabilité, de l'autonomie, de l'intégration et de la participation active à des valeurs.

- L'étude étymologique du mot " éducation " rejoint, en ce sens, celle du mot "pédagogie " du grec "pais", "paidos», " enfant », d'où " paideuein", " élever un enfant " et "paideia", " éducation ", portant les sens à la fois de modelage (formation de la personne) et d'élévation.

Qu'il s'agisse d'éducation ou de pédagogie, deux directions interdépendantes s'articulent donc étymologiquement l'une à l'autre:

- Modeler, produire et reproduire d'un côté;

- Élever vers le « haut » l'élève, l'encourager à s'élever vers sa véritable forme, en d'autres termes, de tendre vers la plus grande authenticité, atteindre la Forme véritable (Gestalt) ou, pour Platon, avoir accès aux réalités intelligibles de la nature humaine considérées comme archétypes et modèles de toutes choses.

2 Jacqueline Pinoche, « Dictionnaire étymologique du français », Ed. Le Robert 1994. 
D’un côté, l'éducation porte sur la " doxa ", les croyances, les identifications, les représentations, les mécanismes et valeurs collectives qu'une société possède pour s'organiser et pour se développer.

De l'autre apparait la nécessité de dynamiser les vertus individuelles, ce que l'on retrouve dans le mythe grec du héros. "L'arété », souvent traduit par " excellence " correspond à celui qui a atteint la plénitude de sa réalisation. Il consiste à avoir le courage et la force de dépasser toutes les adversités afin de réaliser son destin. La recherche d'excellence correspond donc, traditionnellement, aux soins que l'on doit donner à l'âme afin de sortir de la caverne des opinions (doxa) et, atteignant à l'intelligible (gnosis et épistémè), avoir la révélation du sens de sa vie et de sa destinée.

Le jeu dialectique construit par l'étymologie des mots " éducation " ou " pédagogie ", possédant les sens "d'élever » et de "former » va donc bien dans ce sens. Il se retrouve aussi dans le concept classique de Poiésis et de Praxis ${ }^{3}$ fréquemment utilisé dans la philosophie de l'Éducation.

La Poiésis est un acte de production et de reproduction technique (l'art du potier). Il s'agit de la reproduction du même, une forme répétée et, en ce sens, l'action (souvent effectuée par des esclaves ou des catégories sociales basses) importe moins que le but objectif à atteindre. L'objet produit, en éducation, est évalué par des critères déterminés en fonction d'un savoir établi.

La Praxis, inversement, n'a pas de valeur par sa finalité. Elle vaut pour ellemême. Ce qui est pointé, c'est l'homme en tant qu'il est lui-même. C'est l'action et non le produit qui est privilégié et évalué dans une philosophie de la liberté et de l'unicité. Le chemin se fait cheminant, dirigé vers les plus hautes valeurs intellectuelles et éthiques car, pour Aristote, la pensée et l'intelligence ont pour objet la réception de l'Intelligence divine, acte pur de réalisation de soi.

Ces valeurs se sont plus ou moins maintenues jusqu'au XVIème siècle. La notion "d'honnête homme ", au XVIIème siècle, témoigne déjà d'un début de réductionnisme en lequel le sujet singulier devient simplement moral, social et cultivé. Car est apparue, entre le XVIème et le XVIIème siècle, une rupture paradigmatique ${ }^{4}$ niant la part médiatrice de l'âme et dévalorisant la subjectivité. Une seconde suivra, au XIXème siècle, qui construira le paradigme positiviste. Ces deux ruptures ont progressivement valorisé la matérialité au détriment de la spiritualité, l'objectivité au détriment du sujet et de la subjectivité, la reproductibilité et la

3 Aristote., Éthique à Nicomaque, Paris, Le Livre de Poche, trad. Jules Barthélemy-Saint-Hilaire, revue par

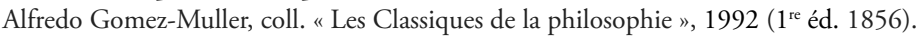

4 Par exemple, voir les travaux de Michel Foucault, «Les mots et les choses : une archéologie des sciences humaines » Ed. Gallimard, Paris, 1966. 
quantité ont effacé l'importance de la singularité et de la qualité, la clôture des objets disciplinaires s'est effectuée au préjudice de leurs interactions, relations et interfaces. L'avoir (matérialisme) a pris le dessus sur l'être (l'ontologique). Un nivellement effaçant progressivement la pluralité des mondes et de la vie humaine a fini par réduire la complexité du réel à un simple monisme matérialiste et existentialiste.

Dans le même mouvement des derniers siècles, la part "élévation » du sujet singulier dans l'éducation s'est logiquement effacée pour se démocratiser et ne plus voir dans ce mot que des " objets ", moules et modèles, ceux de la Poiésis, à enseigner au plus grand nombre.

Lidée d'éducation s'est alors fixée sur une pédagogie limitée à l'ingurgitation, parfois forcée, de nourritures psychiques à visée cognitive, le résultat (la production scientifique, le diplôme...) ayant plus d'importance que la qualité de vie, la félicité des élèves ou la formation véritable des personnes: combien de souffrances vécues dans le système éducatif, combien de diplômés sans emploi ou sans bonheur dans la pratique de leur métier, combien d'inadéquations entre le monde de la formation et celui du travail, combien de conséquences négatives des savoirs et des techniques sur l'environnement, combien peu se valorise l'auto-connaissance!

En même temps, l'éducation moderne ne peut ressortir d'une pensée schizoïde qui viserait à éliminer le pôle de l'ontologie pour valoriser la seule réalité matérielle.

Nous pouvons ainsi imaginer un modèle qui pourrait porter sur une éducation globale, associée à la fois aux nécessités générales ou locales liées aux programmes (currículo), mais aussi à la reconnaissance de la singularité des élèves ou des professeurs à qui ces programmes sont destinés. Ce processus est à la fois formel et informel de même qu'il est temporel, la formation de soi s'effectuant tout au long de la vie.

La démarche transdisciplinaire vise, en ce sens, à réarticuler le sujet et l'objet, les programmes à l'élève, la Poiésis et la Praxis, comme d'ailleurs le Régime diurne du conscient et les Régimes nocturnes de l'inconscient ${ }^{5}$, de façon à intégrer une réalité humaine globale impliquant toutes les dimensions de l'être.

Cette complexité peut devenir une invitation pour penser autrement la trajectoire cognitive (et auto-cognitive) en éducation dans un cadre plus global, celui de l'anthropoformation.

Le néologisme d'anthropoformation que l'on peut traduire par "l'homme en formation " et « la formation de l'homme " (Pineau, 1997) s'enracine dans

5 Ces trois Régimes ont été décrits par Gilbert Durand, "Les structures anthropologiques de l'imaginaire ", Ed. Dunod, Paris, 1969, réed. 1992. Ils ont été repris par G. Pineau dans la notion de trajet anthropoformateur en deux temps et trois mouvements. 
une référence anthropologique explicite et dans une définition supportée par une boucle de récursivité croisée. Elle s'inscrit, en particulier, dans le champ des histoires de vie en posant la question de la construction identitaire de la personne. Cette identité est à la fois consciente et inconsciente. Consciente, parce que nous pouvons appréhender la part existentielle de l'homme qui possède une identité biologique et sociale. Inconsciente, parce qu'une part de l'individu échappe systématiquement à ces identifications. Sur le fond, nous ne savons pas qui nous sommes. En d'autres termes, les questions de l'Être, de l'Ontos et du sujet véritable qui résident de façon voilée en chacun imposent un chemin anthroporévélateur que la formation de soi tout au long de la vie tendra à manifester.

La formation de la personne est un processus vital et permanent qui émerge des différents niveaux qui composent l'identité humaine (à la fois biologique, socioculturelle, intérieure et spirituelle) et avec lesquels il interagit. Ce processus repose sur une vision systémique, un système auto-poïétique et des boucles étranges rétroactives (G. Pineau, 2005): par exemple, l'environnement façonne le sujet qui façonne à son tour son environnement; la société façonne la personne comme cette dernière a pouvoir d'influer sur la société en laquelle elle vit; etc.

Gaston Pineau a développé, dans ses recherches, la théorie tripolaire de la formation: écoformation, hétéroformation, autoformation. Amplifiant les travaux de Gaston Pineau en Sciences de l'Éducation et les appuyant sur la relation entre conscient et inconscient, nous avons avancé un quatrième pôle, ontologique (P. Paul, 2003, trad. Port. 2009):

- Le pôle de l'Écoformation (l'être au monde de la nature) se compose des interactions physiques, climatiques, de la temporalité et des cycles qui mettent en forme et en formation la personne.

- Le pôle de l'Hétéroformation (l'être au monde de la société et de sa culture) inclut toutes les influences familiales, sociales et culturelles qui construisent l'identité sociétale. Nous sommes formés, et même formatés, comme nous formons et formatons les autres par la communication et par l'éducation.

- Le pôle de l'Autoformation (l'être au monde intérieur) est dirigé par le sujet. Toute la question est de savoir de quel sujet l'on parle (P. Paul, 2005, p. 176). Car l'auto-conscience de soi ne peut résumer à la partie vécue, sans présumer du devenir à être, niché au cœur de l'inconscient. Ainsi, de façon rétroactive encore, le sujet conscient, en s'intériorisant, éclaire la réalité du sujet inconscient qui à son tour se révèle à la conscience.

- Le pôle de l'Ontoformation (l'être au monde spirituel) tisse les liens entre le singulier et l'universel, phénoménologie (ou immanence) et transcendance 
(théophanie "affirmative »), ce tissage voilant et révélant tout à la fois les relations entre individualité et universalité .

Dans le cadre transdisciplinaire de l'anthropoformation ${ }^{6}$ associée aux sciences de l'Éducation, il faut entendre :

- La notion d'Écoformation comme l'aspect "production " de l'éducation (impliquant les savoirs corporels, environnementaux, écologiques, expérientiels, tacites). Dans le Rapport Delors ${ }^{7}$ sur les quatre piliers de l'Éducation du XXIème siècle: apprendre à faire, à être au monde.

- L'Hétéroformation développe l'aspect " nutrition " de l'éducation composé des savoirs explicites et académiques essentiellement de l'ordre de la Poiésis et construits sur l'importance des programmes. Elle insiste sur les lois et la morale rendant possible la vie sociale. Rapport Delors: apprendre à vivre ensemble.

- L'Autoformation insiste sur l'aspect " élévation " de l'éducation en relation avec l'émancipation, l'autonomie, l'auto-connaissance, la coopération, la coparticipation, l'intégration des contradictions. Cette dimension tisse le lien entre Praxis et Poiésis, programme et élève, savoir-faire et savoir-être etc. Elle soulève aussi la question de l'éthique. Rapport Delors: apprendre à connaître - ce qui, dans la vision antique, repose toujours sur l'auto-connaissance.

- L'Ontoformation enfin doit se comprendre comme ressortant du tissage entre les différents niveaux de l'éducation/formation tout au long de la vie. Elle développe les liens entre Régimes diurne et nocturne, conscient et inconscient, nature et culture, sciences et traditions, matérialité et spiritualité, etc. Rapport Delors: apprendre à être.

Chacune des catégories constitutives du trajet anthropoformateur obéit, répétons-le, à un processus de boucles récursives. Nous sommes formés par le monde physique, comme nous le façonnons ; nous sommes formés par les autres comme nous les formons ; nous sommes créés par nous-mêmes comme nous créons ce que nous sommes; nous sommes Un dans la diversité de sa manifestation. Il importerait en ce sens de favoriser un modèle éducatif intégral qui

6 PINEAU, Gaston., "Formation expérientielle et théorie tripolaire de la formation ", in La formation expérientielle des adultes, Recherches en Formation continue, La documentation française, Paris, 1991, pp.29-40 et PAUL, P., Formation du sujet et transdisciplinarité, Ed. L'Harmattan, 2003.

7 DELORS, Jacques, 1997: L'éducation - Un trésor est caché dedans. Rapport à l'UNESCO de la Commission internationale sur l'éducation pour le XXIe siècle, présidée par Jacques Delors. 
puisse dépasser les représentations duelles habituelles (distinguer le vrai du faux, évaluer les connaissances et non des personnes, se désintéresser de la dimension vitale, spirituelle et inconsciente qui, cependant, nous sustente etc.). Le savoir devrait pouvoir s'articuler avec le savoir-faire, le savoir-être ${ }^{8}$ et l'être. Au total, articuler la volonté d'objectivité scientifique avec la réalité des sujets et leur subjectivité qui nécessairement participe des observations et de leur connaissance 9 . Les pratiques et savoirs marginalisés mais orientés vers l'auto-connaissance, les savoirs associés à l'environnement et à la qualité de vie devraient aussi être réintégrés dans l'éducation. Cette complexité intégrative et coopérative permettrait sans doute de mieux répondre aux nombreux défis du monde moderne tout en favorisant la félicité individuelle qui apparait sitôt que l'on donne sens et conformité à la vie singulière.

Il convient aussi de reconnaître la pluralité ou, mieux encore, l'unité plurielle sur laquelle repose le monde, la société et chaque individu singulier. Au sein de la pluralité les points du vue séparateurs pouvant se contredire, il convient d'apprendre à réunir les contradictions, ce qui impose d'ouvrir la connaissance à des méthodologies et à une épistémologie qui puisse favoriser sa mise en relation et en résolution. La coopération, l'interactivité, la participation, le dialogue, l'intersubjectivité, la réciprocité devraient en ce sens devenir des valeurs expérimentales et éthiques associées à la méthode. La méthodologie ${ }^{10}$, intégrative des contradictions, accueille et respecte les différences dans une dynamique participative et transformatrice.

La réflexion proposée ici peut être qualifiée de paradigmatique. Mais elle renvoie à d'autres catégories qu'a développé le Laboratoire des Sciences de l'Éducation de l'Université de Tours dirigé par G. Pineau dans les années 2000. Trois catégories ont alors été avancées, associées à la démarche transdisciplinaire (P. Paul, G. Pineau, 2005) considérée selon les cas comme socio-interactive, réflexive ou paradigmatique. En d'autres termes, la théorie des recherches transdisciplinaires en éducation répond à la pratique comme la pratique nourrit la théorie.

L'éducation du futur semble donc appeler l'introduction d'un nouveau

8 Intégrer, par exemple, les connaissances par l'expérience, dynamiser les vertus éthiques des personnes mais aussi savoir dépasser les modèles, voire les transgresser chaque fois que les nécessités vitales imposent de nouveaux défis.

9 PAUL, P., "Importância do sujeito e da subjetividade na epistemologia e na avaliação da interdisciplinaridade ", in Práticas da interdisciplinaridade no ensino e pesquisa, Editores Arlindo Philippi Jr., Valdir Fernandes, Ed. Manole, São Paulo, 2015, pp. 137-164.

10 La méthodologie du tissage en particulier- P. Paul, 2003. 
paradigme, transdisciplinaire ${ }^{11}$, qui puisse créer des ponts entre les disciplines, entre objets et sujets, entre science moderne et traditions millénaires, entre Orient et Occident, entre nature et culture etc. Il suggère, plus précisément, d'insister sur les interfaces qui séparent/relient objectivité et subjectivité, analyse et intégration, conscient et inconscient.

La question de "l'Anthropos ", de l'homme, trop souvent réduite à sa part consciente et existentielle, devrait au final pouvoir se rouvrir à sa réalité plus profonde, vitale, intuitive, créative et inconsciente de façon à clarifier le sens de sa vie dans le monde et dans la société. Éduquer, c'est reconnaître qu'aucune forme effective ne peut provenir seulement de l'extérieur de la personne humaine. La dialectique de l'apparent et du caché, du connu et de l'inconnu rappelle le sens profond de la réalité humaine qui aspire à la fois à une volonté légitime de sécurité - impliquant de valoriser le contrôlable, le mesurable, le quantitatif - mais qui doit aussi accepter l'individuel, le qualitatif, l'imprévisible, la crise qui, en rompant avec l'ordre établi, viennent dynamiser nos transformations et nous rappeler que le trajet éducatif et anthropoformateur se manifeste toujours à l'interface des programmes et de la formation de soi.

\section{Referências}

Paul, P. Pensamento complexo e interdisciplinaridade: abertura para mudança de paradigma? In: Interdisciplinaridade em ciência, tecnologia e inovaçáo. Ed. Manole, 2011, pp. 229-259.

Paul, P; Pineau, G. Transdisciplinarité et formation. Ed. L'Harmattan, 2005.

Pineau, G. Nouvel esprit anthropologique en reconnaissance et validation des acquis. In: Revue Paroles et pratiques sociales, $n^{\circ}$ 54/55, 1997.

Thomas S. Kuhn, La structure des révolutions scientifiques. Paris Flammarion, Coll. Champs-Sciences, 2008 ( $1^{\text {re }}$ éd. 1962) 